\title{
Special Issue on Probabilistic Structural Dynamics and Earthquake Engineering
}

\author{
FOREWORD
}

Sources of uncertainty in structural dynamics are traceable to modelling errors as well as inherent imperfections in structural geometry and excitations, among others. Given that the most acceptable route towards a rational description and modelling of this uncertainty is through the use of probability theory and stochastic processes, new advances in the theory and applications to stochastic structural dynamics assume great significance. This is particularly true, for instance, in the broad field of earthquake engineering owing to the well-researched and widely appreciated stochastic models for earthquake excitations. Earthquake resistant structures are designed to display controlled inelastic behaviour, and added to this need to model the nonlinear behaviour of structural systems, stochastic modelling naturally adds to the complexity of analysis and thus poses a challenge to researchers. Indeed, for most stochastically driven nonlinear systems of engineering interest, exact analytical solutions are hardly available and the analyst has to fall back on either a clever analytical approximation or a direct numerical simulation. Approximate analytical techniques, unfortunately, have not been widely successful in accurately predicting the response of strongly nonlinear and higher dimensional systems. Therefore the research focus has, to an extent, veered round to the development and exploration of novel forms of simulation tools, e.g. Monte Carlo simulations using numerical integrations. Here again, developments of accurate schemes for stochastic numerical integrations face many more challenges than such developments in the context of deterministic systems. Even from an intuitive viewpoint, a stochastic trajectory would generally have more variations over a given length than its deterministic counterpart. In order to allow for this, such a trajectory cannot be modelled as $C^{\infty}$, i.e., the modelling has to be consistent with the fact that it is differentiable only a limited number of times. In order to account for such non-smoothness, different calculi (such as Ito, Stratonovich or Malliavin calculi) have been developed. Of these, Ito calculus has been particularly useful for developing approximate analytical and numerical tools in stochastic structural dynamics. Over the last few decades, these analytical and numerical tools have been exploited to solve problems in many different areas of engineering relevance, as reflected partly in the papers published here.

This special issue consists of eleven papers reflecting research progresses in some of the broad areas on stochastic structural dynamics and applications to earthquake engineering. The topics covered include random eigenvalue problems through an asymptotic expansion of multi-dimensional integrals, Monte Carlo simulations of an ecosystem with two competing species, stochastic averaging applied to determining the PDF of strongly nonlinear dynamical systems, a study on explicit dependence of strength reduction factors of elasticplastic oscillators on ground motion parameters, combining stochastic finite element with the response surface method for estimating structural reliability, nonlinear dynamics of large deformations of a beam sliding on two knife-edge supports, particle filters applied to the identification of nonlinear structural dynamical systems, importance sampling in time-varying 
reliability analyses, improved damage indicators through a multi-resolution analysis, and stochastic analysis of random road profiles on vehicle dynamics.

We would like to thank all the authors who have contributed to this special issue. We would also express appreciation for the timely reviews provided to the authors by the experts. In addition, we thank the Editor, Sādhana and the Indian Academy of Science, for providing the forum for the dissemination of this work.

August 2006

D ROY

C S MANOHAR

Guest Editors 